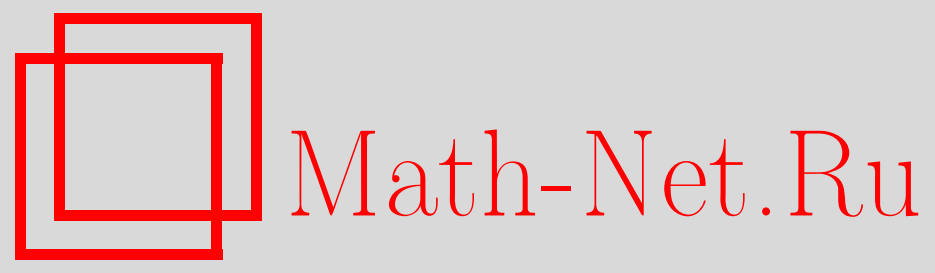

Х. А. Мадатян, О полных проверяющих тестах для квазибесповторных контактных схем, Дискрет. матем., 1996, том 8, выпуск 3, 111-118

DOI: https://doi.org/10.4213/dm535

Использование Общероссийского математического портала Math-Net.Ru подразумевает, что вы прочитали и согласны с пользовательским соглашением http://www . mathnet.ru/rus/agreement

Параметры загрузки:

IP : 54.166 .219 .16

26 апреля 2023 г., 16:54:19 


\title{
О полных проверяющих тестах для квазибесповторных контактных схем
}

\author{
(C) 1996 г. Х. А. Мадатян
}

При әксплуатации сложных логических схем особую важность приобретает вопрос о контроле работы әтих устройств, о способах отыскания повреждений в них. Для контроля логических устройств важное значение имеет построение минимальных тестов, при помощи которых можно не только определить, исправна или нет схема, но и указать вид и место возникшей неисправности. Однако во многих случаях практическое построение тестовых процедур является весьма трудоемким. В настоящей статье рассмотрены отдельные классы схем, для которых построение тестов упрощается в связи с особенностями схемы.

Работа выполнена при поддержке Грантового центра по исследованиям в области математики при Новосибирском государственном университете (проект «Математические вопросы синтеза надежных и легкотестируемых схем»).

\section{Введение}

При әксплуатации сложных логических схем особую важность приобретает вопрос о контроле работы этих устройств, о способах отыскания повреждений в них. Для контроля логических устройств важное значение имеет построение минимальных тестов [1], при помощи которых можно не только определить, исправна или нет схема, но и указать вид и место возникшей неисправности.

Математический аппарат построения тестовых процедур разработан в статье А. И. Чегис и С. В. Яблонского [1]. Однако, во многих случаях практическое использование этого алгоритма затруднено из-за большой его трудоемкости. Поэтому естественно рассматривать отдельные классы схем, для которых построение тестов упрощается в связи с особенностями схемы.

Пусть контактная схема $\Sigma$ реализует функцию алгебры логики $f\left(x_{1}, \ldots, x_{n}\right)$. Предположим, что в схеме $\Sigma$ при воздействии источника помех возникают какието неисправности, при которых схема $\Sigma$ переходит в некоторую схему $\Sigma \prime$. Обозначим через $f^{\prime}\left(x_{1}, \ldots, x_{n}\right)$ проводимость схемы $\Sigma^{\prime}$. Функция $f^{\prime}\left(x_{1}, \ldots, x_{n}\right)$ называется функцией неисправностей. Множество всех функций неисправностей разбивается на классы $F_{0}, F_{1}, \ldots, F_{m}$ такие, что $f\left(x_{1}, \ldots, x_{n}\right) \in F_{0}$ и функции $f^{\prime}$ и $f^{\prime \prime}$ относятся к одному и тому же классу тогда и только тогда, когда

$$
f^{\prime}\left(x_{1}, \ldots, x_{n}\right) \equiv f^{\prime \prime}\left(x_{1}, \ldots, x_{n}\right) .
$$


Типичными для контактных схем являются два вида неисправностей: короткое замыкание и разрыв контакта. Мы будем предполагать, что источник помех воздействует дискретно. Контроль ведется путем экспериментов без вмешательства в схему, т.е. на входы схемы подают любые наборы и наблюдают значения проводимости. Для того, чтобы иметь возможность анализировать состояние схемы после очередной неисправности, мы предполагаем, что при выполнении эксперимента источник помех не действует.

Совокупность входных наборов схемы $\Sigma$ называется проверяющим тестом $\left(\mathrm{T}_{\mathrm{n}}\right)$ относительно заданного перечня неисправностей, если по значениям проводимости схемы на этих наборах можно судить об исправности схемы.

Очевидно, что $\mathrm{T}_{\mathrm{n}}$ дает возможность отличить класс $F_{0}$ от $F_{1} \cup \ldots \cup F_{m}$.

Длиной теста будем называть число наборов, входящих в тест.

С точки зрения допустимых неисправностей рассматриваются единичные и полные тесты.

Единичный тест - это тест, предназначенный для обнаружения неисправностей, когда заранее известно, что неисправность допустима для единственного контакта.

Полный тест - это тест для обнаружения неисправностей, когда неисправности (замыкания и разрывы) допускаются для любого подмножества контактов схемы.

Назовем неисправностью типа $\mathrm{H}_{1}$ такую неисправность, при которой все неисправные контакты разомкнуты.

Неисправностью типа $\mathrm{H}_{2}$ назовем неисправность, при которой все неисправные контакты замкнуты.

Неисправностью типа $\mathrm{H}_{3}$ назовем неисправность, при которой часть неисправных контактов замкнута, а часть разомкнута.

Легко показать, что если Т - единичный проверяющий тест для схемы $\Sigma$, то $\mathrm{T}$ обнаруживает также все неисправности типа $\mathrm{H}_{1}$ и $\mathrm{H}_{2}$.

Определим теперь некоторые классы схем [2].

Класс $A_{\&}^{0}=A_{\vee}^{0}$ состоит из контактных схем, содержащих по одному контакту. Класс $A_{\vee}^{k}$ определяется следующим образом.

(1) $A_{\&}^{k-1} \subseteq A_{\vee}^{k}$.

(2) Если $\Sigma_{1} \in A_{V}^{k}, \Sigma_{2} \in A_{V}^{k}$, а схема $\Sigma$ получается параллельным соединением схем $\Sigma_{1}$ и $\Sigma_{2}$, то $\Sigma \in A_{\vee}^{k}$.

(3) Класс $A_{\vee}^{k}$ не содержит никаких других схем.

Класс $A_{\&}^{k}$ определяется двойственным образом.

Схема $\Sigma$ называется бесповторной, если каждая переменная приписана не более чем одному контакту этой схемы.

Контактную схему $\Sigma$ будем называть квазибесповторной, если каждый из символов $x_{i}, \bar{x}_{i}$ приписан не более чем одному ребру.

Схему назовем тупиковой, если при размыкании или замыкании любого числа контактов функция проводимости изменяется. В дальнейшем все схемы будем предполагать тупиковыми, не оговаривая это дополнительно.

Тесты для бесповторных схем исследовались в работах [3-5]. В [3] строится полный проверяющий тест для бесповторных схем, сложность которого не пре- 
восходит $n+1$, где $n$ - число контактов в схеме. Для бесповторных $\pi$-схем в [5] получено точное значение длины минимального проверяющего теста.

$\mathrm{B}$ данной работе найдено точное значение функции Шеннона для полного проверяющего теста квазибесповторных схем из класса $A_{\vee}^{2}\left(A_{\&}^{2}\right)$. Для квазибесповторных $\pi$-схем доказано, что функция Шеннона для полных проверяющих тестов линейно зависит от числа переменных.

\section{1. Построение проверяющих тестов для квазибесповторных схем классов $A_{\vee}^{2}$ и $A_{\&}^{2}$}

Пусть схема $\Sigma$ реализует функцию $f\left(x_{1}, \ldots, x_{n}\right)$ и имеет сложность $L(\Sigma)$.

Определение. Набор $\tilde{\alpha}$ называется проверяющим для контакта $x$ сжемы $\Sigma$ (проверяет контакт $x$ в схеме $\Sigma$ ) если при неисправности (замыкании или размыкании) одного этого контакта выполняется условие

$$
f^{\prime}(\tilde{\alpha}) \oplus f(\tilde{\alpha})=1
$$

где $f^{\prime}-$ функция неисправности.

Предложение. Множсество наборов $E$ образует единичный проверяющий тест замыкания (размыкания) тогда и только тогда, когда для кахсдого контакта $в$ мнохсестве Е найдется хотя бы один проверяющий набор.

Лемма 1. Для произвольной схемы $\Sigma$ из класса $A_{\vee}^{2}$ (соответственяо $A_{\&}^{2}$ ) любой единичный проверяющий тест является полным проверяющим тестом.

Доказательство. Пусть схема $\Sigma$ принадлежит классу $A_{\vee}^{2}$ и реализует функцию $f\left(x_{1}, \ldots, x_{n}\right)$, а $\Sigma^{\prime}$ - неисправная схема и $f^{\prime}-$ ее функция проводимости. Очевидно, что если неисправность схемы является неисправностью типа $\mathrm{H}_{1}$ или $\mathrm{H}_{2}$, то единичный тест проверяет ее. Рассмотрим случай неисправности $\mathrm{H}_{3}$. Пусть $x_{i}^{\sigma}$ - один из разомкнутых контактов. В единичном тесте есть набор $\tilde{\alpha}=\left(\alpha_{1}, \ldots, \alpha_{n}\right)$, проверяющий контакт $x_{i}^{\sigma}$ на размыкание.

Если $f^{\prime}\left(\alpha_{1}, \ldots, \alpha_{n}\right)=0$, то на наборе $\tilde{\alpha}$ неисправность проверяется.

Предположим, что $f^{\prime}\left(\alpha_{1}, \ldots, \alpha_{n}\right)=1$. Это означает, что в схеме $\Sigma^{\prime}$ имеется цепь $z$ с ненулевой проводимостью на наборе $\tilde{\alpha}$, содержащая хотя бы один неисправный замкнутый контакт $x_{j}^{\delta}$. В единичном тесте существует набор $\tilde{\beta}=\left(\beta_{1}, \ldots, \beta_{n}\right)$, проверяющий этот контакт $x_{j}^{\delta}$ на замыкание. Так как в цепи $z$ нет разомкнутых контактов, то $f^{\prime}(\tilde{\beta})=1$, т.е. набор $\tilde{\beta}$ проверяет неисправность.

Лемма доказана.

Следствие 1. Пусть $\mathrm{T}_{\mathrm{n}}^{1}(\Sigma), \mathrm{T}_{\mathrm{n}}^{\mathrm{n}}(\Sigma)$ - соответственно длины минимального единичного и полного проверяющего тестов для схем из класса $A_{\vee}^{2}\left(A_{\&_{L}}^{2}\right)$. Тогда $\mathrm{T}_{\mathrm{n}}^{1}(\Sigma)=\mathrm{T}_{\mathrm{n}}^{\mathrm{n}}(\Sigma)$. 


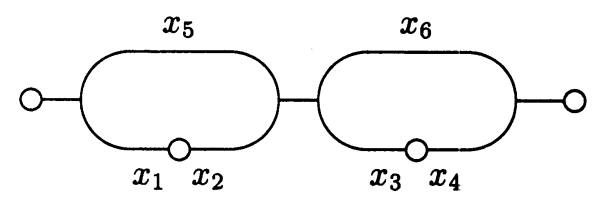

Рис. 1.

Приведем пример бесповторной схемы $\Sigma$ из класса $A_{\&}^{3}$, у которой существует единичный проверяющий тест, не являющийся полным. Рассмотрим схему, приведенную на рис. 1.

Очевидно, что наборы

$$
\begin{array}{lll}
B_{1}=(100111), & B_{2}=(111100), & B_{3}=(011101) \\
B_{4}=(100001), & B_{5}=(000110), & B_{6}=(001010)
\end{array}
$$

образуют единичный проверяющий тест.

Пусть теперь контакты $x_{2}, x_{3}$ замкнуты, а $x_{5}, x_{6}$ разомкнуты. Непосредственной проверкой убеждаемся, что

$$
\begin{aligned}
& f^{\prime}\left(B_{1}\right)=1, \quad f^{\prime}\left(B_{2}\right)=1, \quad f^{\prime}\left(B_{3}\right)=0, \\
& f^{\prime}\left(B_{4}\right)=0, \quad f^{\prime}\left(B_{5}\right)=0, \quad f^{\prime}\left(B_{6}\right)=0 \text {, }
\end{aligned}
$$

т.е. множество наборов $B_{1}, \ldots, B_{6}$ не проверяет әтой неисправности, и, следовательно, не является полным проверяющим тестом.

Отсюда следует, что для классов $A_{\&}^{k}$ и $A_{\vee}^{k}, k \geqslant 3$, утверждение леммы не справедливо.

Пусть $\Sigma$ - произвольная схема из класса $A_{\mathrm{V}}^{2}$, и $\alpha$ и $\beta$ - любые две цепи әтой схемы. Обозначим через $u$ и $v$ произвольные контакты, принадлежащие цепям $\alpha$ и $\beta$ соответственно.

Лемма 2. Контакты $и$ u $v$ невозможсно проверить на замыкание оджим набором тогда и только тогда, когда существуют переменные $x, y, z$ такие, что контакты $x^{\sigma}, y^{\delta}, z^{\gamma}$ принадлехсат $\alpha$, а контакты $x^{\bar{\sigma}}, y^{\delta}, z^{\bar{\gamma}}$ принадлехсат $\beta$.

Доказательство. Справедливость утверждения леммы 2 следует из того, что ни один проверяющий набор не может одновременно проверять контакты из $\alpha$ и $\beta$, когда в $\alpha$ и $\beta$ есть три противоположных контакта. В случае, когда противоположных контактов не более двух, всегда можно построить проверяющий набор для двух контактов этих цепей.

Пусть $\mathrm{T}_{n}^{\mathrm{n}}(\Sigma)$ - длина минимального полного проверяющего теста для схемы $\Sigma$. Введем функцию Шеннона длины полного проверяющего теста для квазибесповторных схем класса $A_{\vee}^{2}$ :

$$
\mathrm{T}_{\mathrm{n}}^{\mathrm{n}}(n)=\max _{\Sigma \in A_{\mathrm{V}}^{2}} \mathrm{~T}_{\mathrm{n}}^{\mathrm{n}}(\Sigma)
$$


Теорема 1. Справедливо равенство

$$
\mathrm{T}_{\mathrm{n}}^{\mathrm{n}}(n)=2 n+[\sqrt{2 n / 3+1 / 4}+1 / 2] .
$$

Доказательстөо. Покажем сначала, что $\mathrm{T}_{\pi}^{\mathrm{n}}(n) \leqslant 2 n+[\sqrt{2 n / 3+1 / 4}+1 / 2]$.

Опишем алгоритм построения теста.

Шаг 1. В схеме выбирается максимальная по длине цепь. В силу тупиковости схемы существует набор $\tilde{\alpha}$ такой, что на нем замкнута только эта цепь. Набор $\tilde{\alpha}$ включается в тест.

Шаг 2. Для каждого контакта рассматриваемой цепи строится набор, проверяющий этот контакт на замыкание. Если таких наборов несколько, то выбирается тот, который проверяет на замыкание максимальное количество ранее не проверенных контактов схемы.

Шаг 3. Если в схеме есть проверенный на замыкание контакт, такой, что цепь, проходящая через него, не проверена на размыкание, то соответствующий әтой проверке набор $\tilde{\beta}$ заносится в тест и выполняется шаг 2 . В противном случае выполняется шаг 1.

Алгоритм продолжает работу, пока в схеме остаются непроверенные контакты.

Пусть $L-$ число обращений к шагу 1 . Очевидно, что длина теста не превосходит

$$
L+r_{1}^{1}+\ldots+r_{k_{1}}^{1}+r_{1}^{2}+\ldots+r_{k_{2}}^{2}+\ldots+r_{k_{L}}^{L},
$$

где $k_{i}$ - число цепей, выбранных в промежутке от $i$-го до $(i+1)$-го обращения к шагу $1, r_{j}^{i}$ - длина $j$-ой такой цепи. Так как соответствующая цепь содержит по три отрицания переменных по крайней мере для $L-1$ цепи, то по лемме 2 $r_{1}^{L} \geqslant 3(L-1)$. Но тогда и подавно

$$
r_{1}^{1} \geqslant 3(L-1), \quad r_{1}^{2} \geqslant 3(L-1), \quad r_{1}^{L} \geqslant 3(L-1) .
$$

Следовательно, в схеме содержится по крайней мере $3 L(L-1)$ контактов. Отсюда следует, что $3 L(L-1) \leqslant 2 n$. Разрешая неравенство относительно $L$, получим, что

$$
L \leqslant \sqrt{2 n / 3+1 / 4}+1 / 2 .
$$

Очевидно также, что $\sum_{i=1}^{L} \sum_{j=1}^{k_{i}} r_{j}^{i} \leqslant 2 n$. Отсюда и из леммы 1 следует, что $\mathrm{T}_{n}^{\mathrm{n}}(n) \leqslant 2 n+[\sqrt{2 n / 3+1 / 4}+1 / 2]$.

Покажем, что

$$
\mathrm{T}_{\mathrm{n}}^{\mathrm{n}}(n) \geqslant 2 n+[\sqrt{2 n / 3+1 / 4}+1 / 2] \text {. }
$$

Выберем сеть $S$, содержащую ровно $[\sqrt{2 n / 3+1 / 4}+1 / 2]$ цепей, такую, что, приписывая ребрам переменные $x_{i}$, можно получить схему из класса $A_{V}^{2}$.

Ребрам первой цепи припишем любые $K=3[\sqrt{2 n / 3+1 / 4}-1 / 2]$ переменные без отрицаний. 
Трем ребрам второй цепи припишем отрицания трех из $K$ переменных, приписанных ребрам первой цепи; трем ребрам третьей цепи припипем отрицания трех из оставшихся $K-3$ переменных, приписанных ребрам первой цепи, и т.д. Оставшимся ребрам второй цепи припишем любые $K-3$ из оставшихся $n-K$ переменных без отрицаний.

Трем из $K-3$ ребрам третьей цепи припишем отрицания трех из $K-3$ переменных, приписанных ребрам второй цепи. Трем из $K-3$ ребер четвертой цепи припишем отрицания трех из оставшихся $K-6$ переменных, приписанных ребрам второй цепи, и т.д. Аналогичным образом приписываем переменные оставшимся ребрам третьей цепи и всех остальных цепей.

Очевидно, что любые цепи $\alpha$ и $\beta$ содержат переменные $x, y, z$ такие, что $x, y, z$ принадлежат $\alpha$, а $\bar{x}, \bar{y}, \bar{z}$ принадлежат $\beta$. Следовательно, для проверки любого контакта на замыкание нужен свой набор. Eще $[\sqrt{2 n / 3+1 / 4}+1 / 2]$ наборов требуется для проверки схемы на размыкание. Отсюда следует, что $\mathrm{T}_{n}^{\mathrm{n}}(n) \geqslant$ $2 n+[\sqrt{2 n / 3+1 / 4}+1 / 2]$. Теорема полностью доказана.

\section{2. Тесты для квазибесповторных $\pi$-схем}

Через $M(\tilde{\alpha})$ будем обозначать множество замкнутых в схеме контактов, когда на вход подан набор $\tilde{\alpha}$.

Докажем теорему, являющуюся по существу обобщением теоремы 1 работы [3].

Теорема 2. Произвольный контакт $x$ схемы $\Sigma$, реализующей фунхчию $f$, исправен, если существует набор о̃, проверяющий контакт $x$ на замыкание, и набор $\tilde{\beta}$, проверяющий контакт $x$ на размыкание, такие, что

$$
M(\tilde{\alpha}) \supseteq(M(\tilde{\beta}) \backslash\{x\}), \quad f^{\prime}(\tilde{\alpha})=0, \quad f^{\prime}(\tilde{\beta})=1 .
$$

Доказательство. Пусть $x$ разомкнут, $f^{\prime}(\tilde{\alpha})=0, \tilde{\alpha}^{\prime}=\left(\alpha_{1}, \ldots, \bar{\alpha}_{i}, \ldots, \alpha_{n}\right)$, $f^{\prime}\left(\tilde{\alpha}^{\prime}\right)=0$. Но тогда $f^{\prime}(\tilde{\beta})=0$, что противоречит условию теоремы.

Пусть $x$ замкнут, $f^{\prime}(\tilde{\beta})=1, \tilde{\beta}^{\prime}=\left(\beta_{1}, \ldots, \bar{\beta}_{i}, \ldots, \beta_{n}\right), f^{\prime}\left(\tilde{\beta}^{\prime}\right)=1$. Но тогда $f^{\prime}(\tilde{\alpha})=1$, что противоречит условию теоремы.

Теорема доказана.

Будем говорить, что контакты $x$ и $y$ в $\pi$-схеме соединены параллельно (последовательно), если существуют подсхемы $\Sigma_{1}$ и $\Sigma_{2}$ такие, что $x \in \Sigma_{1}, y \in \Sigma_{2}$, $\Sigma_{1}$ и $\Sigma_{2}$ соединены параллельно (последовательно).

Теорема 3. В квазибесповторной $\pi$-схеме для любого контакта можсно построить проверяющие наборы $\tilde{\alpha}$ и $\tilde{\beta}$ так, что они удовлетворяют условиям теоре$\mathcal{M b l} 2$.

Доказательство. Пусть $x_{i}^{\sigma}$ - произвольный контакт. Рассмотрим три случая.

1. Контакты $x_{i}^{\sigma}$ и $x_{i}^{\bar{\sigma}}$ соединены последовательно. Пусть набор $\tilde{\beta}$ проверяет $x_{i}^{\sigma}$ на размыкания. Тогда наборы $\tilde{\alpha}=\left(\alpha_{1}, \ldots, \bar{\alpha}_{i}, \ldots, \alpha_{n}\right)$ и $\tilde{\beta}$ удовлетворяют условиям теоремы 2 . 


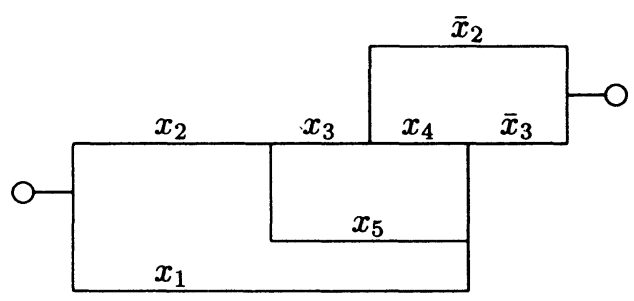

Puc. 2.

2. Контакты $x_{i}^{\sigma}$ и $x_{i}^{\bar{\sigma}}$ соединены параллельно. Пусть набор $\tilde{\alpha}$ проверяет $x_{i}^{\sigma}$ на замыкания. Тогда наборы $\tilde{\beta}=\left(\alpha_{1}, \ldots, \bar{\alpha}_{i}, \ldots, \alpha_{n}\right)$ и $\tilde{\alpha}$ удовлетворяют условиям теоремы 2.

3. В схему входит только один из контактов $x_{i}^{\sigma}$ и $x_{i}^{\bar{\sigma}}$. Тогда наборы $\tilde{\beta}=$ $\left(\alpha_{1}, \ldots, \bar{\alpha}_{i}, \ldots, \alpha_{n}\right)$ и $\tilde{\alpha}$ удовлетворяют условиям теоремы 2.

Теорема доказана.

Следствие 2. Для функции Шеннона көазибесповторных $\pi$-схем справедлива оценка

$$
\mathrm{T}_{\mathrm{n}}^{\pi}(n) \leqslant 4 n
$$

Теорема 4. Для функции Шеннона квазибесповторных $\pi$-схем справедлива оченxa

$$
2 n+n / 3+1 \leqslant \mathrm{~T}_{\pi}^{\pi}(n) \leqslant 4 n .
$$

Доказательство. Достаточно показать, что

$$
\mathrm{T}_{\mathrm{n}}^{\pi}(n) \geqslant 2 n+n / 3+1 \text {. }
$$

Построим схему $\Sigma$ следующим образом.

Рассмотрим дерево с корнем, в котором из каждой вершины, кроме концевых, исходим ровно два ребра.

Ребра, смежные с корнем дерева, будем называть ребрами первого уровня. Ребра, смежные с ребрами $(i-1)$-го уровня, будем называть ребрами $i$-го уровня, если у них нет смежных ребер уровня ниже $i-1$. Пусть построенное дерево имеет $k$ уровней.

Исходя из дерева с корнем, построим схему следующим образом.

Пусть два ребра $i$-го уровня имеют общую вершину. Этим ребрам сопоставим соответственно по три последовательно соединенных контакта $x, y, z$ и $\bar{x}, \bar{y}$, $\bar{z}$.

Очевидно, что для любых цепей $\alpha$ и $\beta$, связывающих концевые вершины с корнем дерева, сушествуют переменные $x, y, z$ такие, что контакты $x, y, z$ принадлежат $\alpha$, а контакты $\bar{x}, \bar{y}, \bar{z}$ принадлежат $\beta$. Следовательно, для проверки каждого контакта на замыкание нужен свой набор. 
Подсчитаем число цепей, покрывающих схему. Оно равно числу ребер $k$-го уровня. Очевидно, что их число равно $2^{k}$. Число переменных равно $n=3\left(2^{k}-1\right)$, т.е. $2^{k}=n / 3+1$. Следовательно, для проверки всех контактов необходимо $2 n+n / 3+1$ наборов.

Теорема доказана.

Приведем пример тупиковой квазибесповторной схемы, не удовлетворяющей условию теоремы 3.

Рассмотрим схему, приведенную на рис. 2. Очевидно, что контакт $x_{3}$ проверяется на замыкание единственным набором $\tilde{\alpha}=(01010)$. На размыкание контакт $x_{3}$ также проверяется единственным набором $\tilde{\beta}=(10101)$.

Легко видеть, что $\neg\left(M(\tilde{\alpha}) \supseteq\left(M(\tilde{\beta}) \backslash\left\{x_{3}\right\}\right)\right)$, что противоречит условию теоремы 3.

\section{Список литературы}

1. Чегис И. А., Яблонский С. В. Логические способы контроля работы әлектрических схем. Труды МИАН СССР им. В. А. Стехлова (1958) 51, 270-362.

2. Лупанов О. Б. О реализации функций алгебры логики формулами из конечных классов. Проблемы кибернетики (1961) 6, 5-14.

3. Коган И. В. О тестах для бесповторных контактных схем. Проблемы кибернетики (1964) 12, 39-44.

4. Ваксов В. В. О тестах для бесповторных контактных схем. Аөтоматика и телемеханика (1965) 26, №3, 521-524.

5. Мадатян Х. А. Полный тест для бесповторных контактных схем. Проблемь кибернетики (1970) 23, 103-118.

Статья поступила 27.12.94. 